\title{
CONTRIBUCIÓN DEL ESTADO DE ZACATECAS (MÉXICO) A LA CONSERVACIÓN DE LA RIQUEZA FLORÍSTICA DEL DESIERTO CHIHUAHUENSE
}

\author{
José de Jesús BaLlezA ${ }^{1}$ y José Luis VillaseñoR ${ }^{2}$ \\ ${ }^{1}$ Universidad Autónoma de Zacatecas, Unidad Académica de Agronomía, \\ km 15.5 carretera Zacatecas-Guadalajara, 98171 Zacatecas, Zacatecas, México. \\ ballezac@yahoo.com \\ ${ }^{2}$ Universidad Nacional Autónoma de México, Instituto de Biología, \\ Departamento de Botánica; Apdo. postal 70-367, 04510 México, D.F., México. \\ vrios@ibiologia.unam.mx
}

\section{RESUMEN}

El Desierto Chihuahuense ( $\mathrm{DCH})$ es la zona árida más extensa y más rica florísticamente de las regiones secas del norte de México. El DCH forma parte de los territorios de siete estados del país, incluyendo Zacatecas que junto con San Luis Potosí representan su extremo sur. Zacatecas contiene alrededor de $46 \%$ de la flora vascular del DCH y la familia Asteraceae es un miembro importante de esta riqueza, tanto a nivel de todo el DCH como en su fracción en la entidad. Desafortunadamente, no se ha reconocido el valor del mencionado estado en las estrategias de conservación de la biodiversidad propuestas hasta ahora para la protección de la flora del DCH. En este trabajo se hace una selección de sitios considerados importantes para la conservación de la riqueza florística del DCH en Zacatecas, utilizando 198 especies de Asteraceae como variable substituta de la diversidad vegetal total y una división de la porción del DCH en el estado en 26 cuadros de 30 minutos de latitud y longitud. La selección de sitios se realizó mediante la aplicación de un método iterativo que indicó la existencia de 18 cuadros que son importantes para la protección de $100 \%$ de las especies de esta familia. Se discute la ubicación de los cuadros más importantes identificados con este procedimiento así como la importancia de Zacatecas para la conservación de la biodiversidad del DCH.

Palabras clave: Asteraceae, conservación, Desierto Chihuahuense, riqueza florística, Zacatecas. 


\section{ABSTRACT}

The Chihuahuan Desert (DCH) is the largest and floristically richest arid zone in northern Mexico. The DCH includes territories of seven Mexican states, including Zacatecas and San Luis Potosí where its southern limit is located. Zacatecas harbors $46 \%$ of the vascular flora recorded in the DCH and the Asteraceae family is an important component of such richness, both throughout the DCH as a whole and within its Zacatecan portion. Unfortunately, Zacatecas has not been adequately considered in the different conservation strategies proposed to protect the floristic richness of the DCH. In this paper we describe a selection of sites in Zacatecas that are important for the conservation of floristic richness, using 198 species of Asteraceae as a surrogate of the entire flora and dividing the fraction of the $\mathrm{DCH}$ in the state into 26 squares of 30 minutes latitude and longitude. The sites were identified by means of an iterative method, the results of which indicate the necessity of protecting 18 squares to ensure the conservation of the totality of species. The location of the most important squares identified, as well as the role that Zacatecas may potentially play in the conservation of the biodiversity of the DCH are discussed.

Key words: Asteraceae, Chihuahuan Desert, conservation, floristic richness, Zacatecas.

\section{INTRODUCCIÓN}

El Desierto Chihuahuense ( $\mathrm{DCH})$ es la zona árida y semiárida más extensa del norte de México. El nombre de este desierto se remonta a 1843, cuando R. B. Hinde habló vagamente de una "Región Chihuahuense" (Morafka, 1977); sin embargo, se empezó a denominar propiamente DCH a partir de la década de 1940, habiendo utilizando este término Shreve (1942) y Dice (1943, citado por Morafka, 1977), entre otros. Se han hecho diferentes propuestas para delimitar su superficie (Shreve, 1942; Contreras, 1955; Johnston, 1974; Morafka, 1977; Schmidt, 1979; Medellín-Leal, 1982; Anónimo, 2004; Anónimo, 2008). De acuerdo con la propuesta de Johnston (1974), el DCH tiene una superficie de alrededor de 505,000 km², en la República Mexicana ocupa parte de los estados de Chihuahua, Coahuila, Durango, Nuevo León, San Luis Potosí, Tamaulipas y Zacatecas, y se extiende hacia Texas y Nuevo México en los Estados Unidos de América. En la propuesta del Instituto Nacional de Estadística Geografía e Informática (INEGI) (Anónimo, 2008) se con- 
sidera que el DCH en México incluye una superficie de aproximadamente 324,978 $\mathrm{km}^{2}$ distribuida en siete entidades federativas (Cuadro 1).

Cuadro 1. Estados de la República Mexicana que incluyen en su territorio a porciones del Desierto Chihuahuense y la diversidad de la flora vascular correspondiente. Se indica la superficie total del estado (ST) y la superficie del estado (SDCH) que forma parte del Desierto Chihuahuense (Anónimo, 2008). Los datos de riqueza total de especies de plantas vasculares y de Asteraceae se refieren exclusivamente a la parte del DCH en el estado (Villaseñor, datos no publicados).

\begin{tabular}{lrrcc}
\hline & $\mathrm{ST}\left(\mathrm{km}^{2}\right)$ & $\mathrm{SDCH}\left(\mathrm{km}^{2}\right)$ & Especies en total & Especies de Asteraceae \\
\hline Chihuahua & 247,455 & 103,686 & 2248 & 381 \\
Coahuila & 151,563 & 110,973 & 2417 & 405 \\
Durango & 123,451 & 27,820 & 1930 & 361 \\
Nuevo León & 64,220 & 15,355 & 2103 & 345 \\
San Luis Potosí & 60,983 & 33,892 & 2008 & 349 \\
Tamaulipas & 80,175 & 3,291 & 1795 & 293 \\
Zacatecas & 75,539 & 29,961 & 1672 & 348 \\
\hline Total & 803,386 & 324,978 & 3900 & 682 \\
\hline
\end{tabular}

Se han sugerido diferentes subdivisiones del DCH (Morafka, 1977; Hernández y Bárcenas, 1996; Anónimo, 2004). La porción sur del DCH (Región del Salado sensu Morafka, 1977; Meseta Central sensu Anónimo, 2004) incluye principalmente a los estados de San Luis Potosí y Zacatecas, que en conjunto suma aproximadamente $63,853 \mathrm{~km}^{2}$ (19.6\% de la porción mexicana del DCH, Cuadro 1). Algunos autores consideran que esta región no forma propiamente parte del DCH. Por ejemplo, Schmidt (1979) propuso excluirla del verdadero DCH porque difiere en su índice de aridez (Van Devender, inédito), mientras que la Comisión Nacional para el Conocimiento y Uso de la Biodiversidad (Anónimo, 1999) la consideró una ecorregión diferente del DCH con base en sus comunidades vegetales y características topográficas y climáticas, denominándola Desierto del Norte de la Meseta Central. Sin embargo, Morafka (1977) encontró elementos para incluirla como parte del DCH, con base en patrones de distribución geográfica de la herpetofauna. Medellín-Leal (1982) también consideró a esta región como parte del DCH, tomando en cuenta aspectos como la distribución geográfica de algunas especies de plantas y animales, tipos de suelo, así como datos climáticos y topográficos; 
además, este autor propuso extender al DCH hasta las zonas áridas y semiáridas de Hidalgo y Querétaro.

El DCH destaca como la zona árida y semiárida del norte de México con mayor riqueza florística. Henrickson y Johnston (inédito) citan 3382 especies de plantas vasculares en esta región; esta cifra es superior a las 2634 especies reportadas para el Desierto Sonorense, que incluye al desierto cálido de Sonora y la península de Baja California (Wiggins, 1964), a las 2086 especies registradas en el Desierto de Baja California, o a las 1400 especies registradas en la zona árida y semiárida de la Planicie del Sur de Texas (Anónimo, 2008; Villaseñor, datos no publicados); esta última ecorregión corresponde casi en su totalidad a la provincia florística de la Planicie Costera del Noreste propuesta por Rzedowski (1978). Además, el DCH sobresale como el sitio con mayor diversidad de especies de la familia Cactaceae del mundo (Hernández et al., 2004).

El DCH está considerado también como un centro importante de evolución de la flora de México debido a su alto número de taxones endémicos (Rzedowski, 1978). Autores como Johnston (1974) y Rzedowski (1978) han señalado que la región contiene alrededor de 1000 especies y 16 géneros de plantas vasculares endémicas de su territorio. Muchas de estas especies son de amplia distribución en el DCH (Fig. 1), como Acacia glandulifera S. Watson, Coryphantha poselgeriana (A. Dietr.) Britton \& Rose, Gaillardia comosa A. Gray, Nicolletia edwardsii A. Gray, Sartwellia puberula Rydb. o Sericodes greggii A. Gray. Sin embargo, otras están asociadas a condiciones edáficas particulares y muestran una distribución geográfica restringida, como por ejemplo Argemone turnerae A.M. Powell, Gaillardia powellii B.L. Turner, G. gypsophila B.L. Turner, Isocoma gypsophila B.L. Turner, Meiomeria stellata (S. Watson) Stand1., Sartwellia mexicana A. Gray, Strotheria gypsophila B.L. Turner, Thelesperma scabridulum S.F. Blake o Varilla mexicana A. Gray, entre otras (Powell y Turner, 1974). La flora del DCH también destaca por presentar la variedad más amplia de formas de vida que exhibe la flora de México (Rzedowski, 1991).

El DCH ocupa la mayor parte de la Altiplanicie Mexicana, la provincia florística más extensa de la Región Xerofítica Mexicana (Rzedowski, 1978). De acuerdo con Rzedowski (1991), la flora fanerogámica de la Región Xerofítica Mexicana está representada por alrededor de 6000 especies $(27 \%$ de la flora total, estimada en 22,000 especies por el autor). Datos más recientes, derivados de una intensa revisión bibliográfica, han permitido documentar la presencia de 6476 especies asociadas a los matorrales xerófilos (Villaseñor, datos no publicados). Con base en esta información, la flora vascular nativa de los matorrales xerófilos ubicados en esta provincia 
Balleza y Villaseñor: Zacatecas en la conservación de la riqueza florística del Desierto Chihuahense

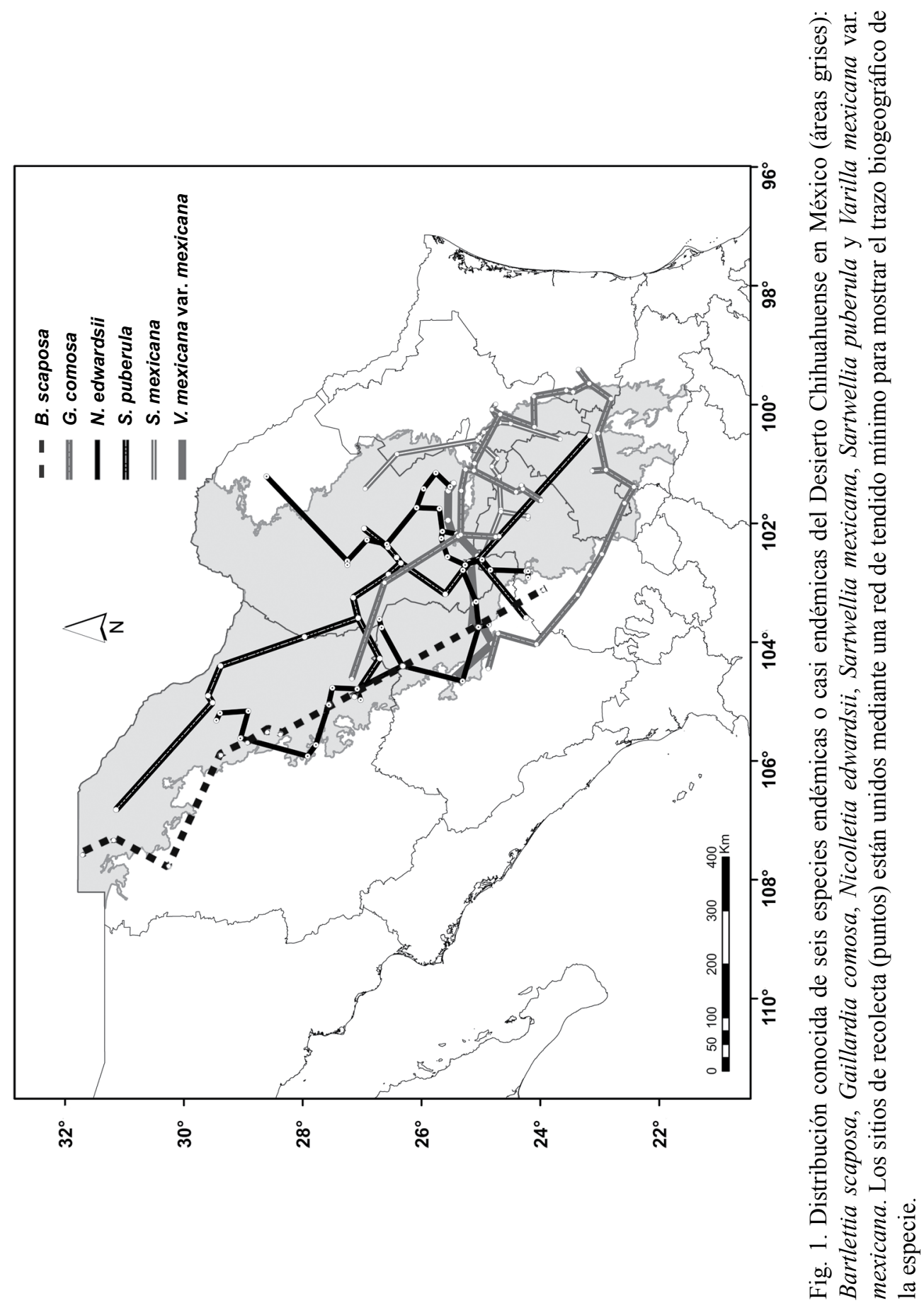


florística del centro y norte de México, se estima en unas 4390 especies, repartidas en 1161 géneros y 170 familias.

El DCH está comprendido en tres de las 19 provincias bióticas definidas por Ferrusquía-Villafranca (1990) con base en rasgos morfotectónicos. Su parte norte está incluida en las provincias Chihuahuense y Coahuilense y la sur en la Altiplanense. La primera está ubicada principalmente, como su nombre lo indica, en el estado de Chihuahua, aunque incluye también el extremo de Durango; la Coahuilense se ubica preponderantemente en territorio del estado de Coahuila, pero también abarca parte de los de Chihuahua, Durango, Nuevo León y Tamaulipas. Finalmente la provincia Altiplanense comprende parte de los estados de Aguascalientes, Coahuila, Durango, Guanajuato, Jalisco, Querétaro, Nuevo León, San Luis Potosí, Tamaulipas así como Zacatecas y es la que más discrepa en sus límites geográficos con las diferentes propuestas de delimitación del $\mathrm{DCH}$, pues a veces se incluyen o se excluyen parte de algunos de los estados que limitan este territorio al sur (Aguascalientes, Guanajuato, Jalisco y Querétaro).

Una evaluación preliminar de la riqueza florística de los matorrales xerófilos, adoptando la circunscripción de las tres provincias bióticas de Ferrusquía-Villafranca (1990), revela la existencia de 3900 especies (Villaseñor, datos no publicados). La provincia Altiplanense registra la mayor diversidad (3100 especies), seguida por la Coahuilense (2621) y la Chihuahuense (1687). De este número, 709 especies han sido reconocidas hasta la fecha como estrictamente endémicas de estas tres provincias (16.3\% del total). La parte sur del DCH (equivalente a la mayor parte de la provincia biótica Altiplanense), incluye principalmente los estados de San Luis Potosí y Zacatecas. Esta región es la más rica florísticamente de todo el DCH, pues de ahí se han registrado hasta la fecha 2610 de las 3100 especies de plantas vasculares conocidas en toda la provincia biótica. La distribución de esta diversidad entre las entidades federativas que incluyen en su territorio parte del DCH se muestra en el Cuadro 1.

La familia Asteraceae se ubica entre las más ricas en la flora de las zonas áridas y semiáridas de México (Rzedowski, 1972; Villaseñor, 1993) y es la de mayor número de especies en la flora vascular del DCH. En el Cuadro 1 se indica el número de especies de Asteraceae que constituyen parte de la flora total conocida en los estados que contienen en su territorio político parte del DCH.

El mayor porcentaje del territorio nacional decretado como área natural protegida (ANP) corresponde a la zona ecológica árida y semiárida (Toledo y Ordóñez, 1998). Sin embargo, ninguna superficie de la porción sur del DCH está incluida en el Sistema Nacional de Áreas Naturales Protegidas (SINAP). En la actualidad solamente $9123.3 \mathrm{~km}^{2}$ del DCH (2.8\% de la parte mexicana del DCH) han sido incorpo- 
rados al SINAP (Anónimo, 2009): el Área de Protección de Flora y Fauna Cañón de Santa Elena, en Chihuahua $\left(2772.1 \mathrm{~km}^{2}\right)$, las Áreas de Protección de Flora y Fauna Maderas del Carmen $\left(2083.8 \mathrm{~km}^{2}\right)$ y Cuatro Ciénegas $\left(843.5 \mathrm{~km}^{2}\right)$ en Coahuila, y la Reserva de la Biosfera Mapimí, en Durango $\left(3423.9 \mathrm{~km}^{2}\right)$. Se han propuesto siete áreas prioritarias para la conservación de las especies de Cactaceae amenazadas en el DCH (Hernández y Bárcenas, 1996), dos de ellas localizadas en la porción sur del DCH (El Huizache, San Luis Potosí, y Jaumave, Tamaulipas), y de entre 125 áreas terrestres de alta prioridad para conservar la biodiversidad del DCH (Anónimo, 2004), cinco se localizan en San Luis Potosí y sólo una en Zacatecas. A nivel estatal, la red de Áreas Naturales Protegidas de esta última entidad federal está integrada en la actualidad por el Parque Nacional Sierra de Órganos (municipio de Sombrerete), el Parque Estatal La Quemada (Villanueva) y la Zona de Preservación Ecológica El Cedral (Ciudad Cuauhtémoc); todas se localizan en la zona de transición entre el DCH y el Piedemonte de la Sierra Madre Occidental y únicamente la primera cuenta con un listado florístico publicado (Enríquez et al., 2003).

Hay evidencias de que el territorio de Zacatecas debería jugar un papel más relevante en la conservación de la diversidad vegetal del DCH. Evaluaciones preliminares sugieren que la porción del DCH correspondiente a este estado presenta una riqueza florística particularmente grande (Cuadro 1) y diferente de la observada en San Luis Potosí. La flora de DCH en Zacatecas está compartida en mayor medida con Durango. Estos antecedentes también destacan la importancia de Zacatecas en la conservación de la diversidad total de plantas vasculares; por ejemplo, Zacatecas ocupa el último lugar por el número total de especies del DCH (Cuadro 1); sin embargo, cuando se evalúan regiones particulares a lo largo del DCH, las zonas que se revelan con la mayor riqueza conocida en la actualidad son las que se ubican alrededor de las capitales de los estados de Coahuila y Zacatecas. Esto seguramente se debe al mayor esfuerzo que se ha hecho para documentar su flora en los últimos años y las facilidades de exploración por la cercanía a centros de investigación.

El objetivo de este trabajo es determinar áreas que destaquen por su riqueza florística como posibles sitios para la conservación de la biodiversidad de la porción zacatecana del DCH, usando la familia Asteraceae como grupo indicador. Se eligió a tal estado porque dispone de un recuento reciente de las Asteraceae que crecen en su territorio (Balleza y Villaseñor, 2002). Además, una regionalización biogeográfica basada en los patrones de distribución de sus especies (Balleza et al., 2005) permitió identificar una región bien definida que corresponde a su zona árida y semiárida que concuerda en gran medida con los límites del DCH reconocidos en el presente trabajo. La familia Asteraceae ha sido un grupo modelo para estimar la 
riqueza florística de México (Rzedowski, 1991; Villaseñor et al., 2007) y para detectar áreas importantes para la conservación de la flora mexicana (Villaseñor et al., 1998). Con esta información se espera proporcionar datos que conduzcan al fortalecimiento del sistema estatal de áreas naturales protegidas de Zacatecas y contribuir a la conservación de la diversidad florística de la porción sur del DCH.

\section{EL DESIERTO CHIHUAHUENSE EN EL ESTADO DE ZACATECAS}

Con base en los límites del DCH propuestos por el INEGI (Anónimo, 2008), su porción zacatecana colinda al norte con Coahuila, al oeste y al sur con la ecorregión Piedemonte de la Sierra Madre Occidental y al este con San Luis Potosí. La región se caracteriza por ubicarse a mayor altitud sobre el nivel del mar, así como por registrar una precipitación mayor y una variación estacional de temperatura menor que el resto del DCH (Morafka, 1977). El área de estudio cubre una superficie de aproximadamente $29,961 \mathrm{~km}^{2}(42 \%$ del territorio de Zacatecas y $9 \%$ de la parte mexicana del DCH); se ubica entre la latitud $22^{\circ} 20^{\prime}-25^{\circ} 09^{\prime} \mathrm{N}$ y la longitud $100^{\circ} 47^{\prime}-$ $103^{\circ} 00^{\prime} \mathrm{O}$. Los matorrales xerófilos característicos de la región son el desértico micrófilo, el desértico rosetófilo y el crasicaule (Rzedowski, 1957).

La parte suroeste de la región de estudio es en promedio la de mayor altitud, pues las porciones planas alcanzan más de $2000 \mathrm{~m}$ s.n.m., mientras que hacia el norte las llanuras están situadas entre 1600 y 1900 m s.n.m. Las serranías internas en general no llegan a grandes alturas: únicamente la Sierra del Astillero, en el norte del estado de Zacatecas, alcanza 3195 m s.n.m. La mayor extensión del DCH en Zacatecas forma parte de la región hidrológica de El Salado, que se caracteriza por la escasez de corrientes permanentes y por incluir en su territorio pequeñas cuencas endorreicas con lagunas intermitentes en sus partes más bajas. En el norte y el oeste, amplias superficies corresponden a la región Nazas-Aguanaval y en el extremo sur, una área pequeña del DCH se ubica en la región hidrológica Lerma-Santiago (Anónimo, 1981).

La zona de estudio está principalmente constituida por cuerpos de rocas metamórficas y sedimentarias marinas de edad Jurásica y Cretácica; las rocas volcánicas del Terciario dominan sólo al oeste, en las zonas aledañas a la Sierra Madre Occidental. La mayor parte de toda la región se encuentra cubierta por sedimentos continentales del Cuaternario (Anónimo, 1981).

El clima de la región se distingue por la escasez e irregularidad de la precipitación, por la coincidencia de la época de lluvia con la temporada caliente del año, por 
la acentuada variación de la temperatura en escala diurna y por la evaporación muy intensa, debido a la fuerte luminosidad, baja presión y humedad atmosférica (Rzedowski, 1957). De acuerdo con García (1987) y la Síntesis Geográfica de Zacatecas (Anónimo, 1981), los climas de la región corresponden al grupo B (secos); el subtipo $\mathrm{BS}_{0}$ (el más seco de los $\mathrm{BS}$ ) predomina en la mayor parte de esta superficie; en orden de extensión prosigue el subtipo $\mathrm{BS}_{1}$ (el menos secos de los $\mathrm{BS}$ ) que se presenta en el norte y en la zona de transición entre el DCH y el Piedemonte de la Sierra Madre Occidental, y el tipo BW (muy seco o desértico) que se encuentra hacia el noroeste.

\section{MATERIALES Y MÉTODO}

La porción del DCH que corresponde a Zacatecas, con base en los limites propuestos por INEGI (Anónimo, 2008), se dividió en 26 cuadros de 30' de latitud por 30 ' de longitud, usando los paralelos y meridianos principales que cruzan el estado (Anónimo, 1981). En promedio, cada cuadro tiene una superficie de $2500 \mathrm{~km}^{2}$ (Fig. 2). Las coordenadas geográficas de los sitios de recolección de los ejemplares de herbario que respaldan las especies incluidas en el Anexo se usaron para ubicarlas apropiadamente. Con estos datos se obtuvo el número total de especies de Asteraceae por cuadro (Cuadro 2).

La flora conocida de Zacatecas incluye 456 especies de Asteraceae (Balleza y Villaseñor, 2002). De esta cifra, 348 se han registrado como miembros de las zonas áridas y semiáridas de México, aunque no todas ellas han sido recolectadas en los matorrales xerófilos de Zacatecas. En este estudio solamente se utilizaron las plantas nativas que fehacientemente han sido recolectadas en la parte del estado que corresponde al DCH (sensu INEGI: Anónimo, 2008), cifra que asciende a 198 especies que se distribuyen en 92 géneros. Las registradas en un solo cuadro se definieron como raras o de distribución geográfica restringida, independientemente de ser endémicas de México o no. Asimismo, los cuadros donde se registraron las especies raras se definieron como sitios irreemplazables para futuros esfuerzos de conservación.

Para seleccionar y asignar prioridades a las áreas potenciales para la conservación de la biodiversidad, los métodos iterativos han sido de los utilizados más ampliamente (Kirkpatrick, 1983; Margules et al., 1988, 1994; Margules y Stein, 1989; Pressey y Nichols, 1989 a, b; Rebelo y Siegfried, 1992; Nichols y Margules, 1993; Pressey et al., 1994; Rebelo, 1994; Turpie, 1995; Ferrier et al., 2000; Margules y Pressey, 2000). Estos procedimientos permiten conservar el mayor número de atributos particulares con interés de conservación en una región determinada usando 


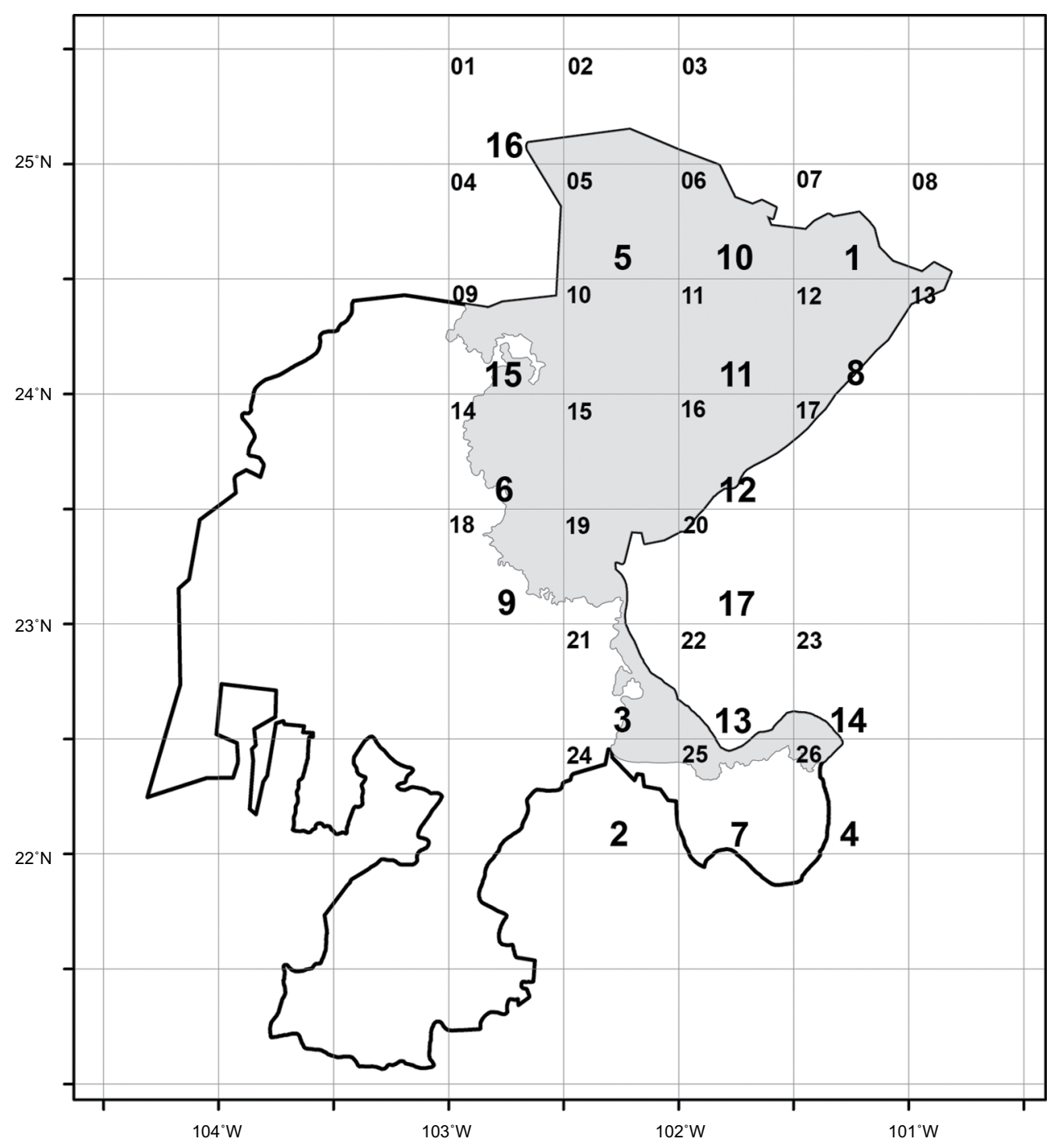

Fig. 2. División de la porción del Desierto Chihuahuense (DCH) en el estado de Zacatecas en cuadros de $30^{\prime}$ de latitud y $30^{\prime}$ de longitud y señalamiento de los cuadros prioritarios para la conservación de la biodiversidad. El número en la esquina superior izquierda es el del cuadro y ubicado en la parte inferior representa el orden de prioridad de conservación de los cuadros mínimos necesarios para la protección de $100 \%$ de las especies de Asteraceae en el estado. 
Cuadro 2. Número de especies de Asteraceae registradas en los cuadros (Fig. 2) en que se dividió la porción de Zacatecas que corresponde al Desierto Chihuahuense (DCH). Se indica el número total de especies por cuadro, el número de especies endémicas de México, de endémicas del DCH y de restringidas (exclusivas al cuadro). Se excluyeron los cuadros que carecen de información.

\begin{tabular}{|c|c|c|c|c|}
\hline Cuadro & Total & Endémicas & Endémicas del DCH & Restringidas \\
\hline 01 & 29 & 8 & 5 & 1 \\
\hline 02 & 1 & 0 & 0 & 0 \\
\hline 04 & 22 & 4 & 4 & 0 \\
\hline 05 & 53 & 17 & 7 & 2 \\
\hline 06 & 67 & 22 & 9 & 2 \\
\hline 07 & 75 & 28 & 12 & 11 \\
\hline 08 & 31 & 8 & 2 & 0 \\
\hline 09 & 34 & 10 & 3 & 1 \\
\hline 10 & 51 & 13 & 8 & 0 \\
\hline 11 & 56 & 13 & 5 & 2 \\
\hline 12 & 63 & 17 & 11 & 3 \\
\hline 13 & 7 & 0 & 0 & 0 \\
\hline 14 & 66 & 20 & 6 & 6 \\
\hline 15 & 61 & 19 & 7 & 0 \\
\hline 16 & 54 & 13 & 5 & 2 \\
\hline 18 & 53 & 14 & 3 & 3 \\
\hline 19 & 54 & 17 & 5 & 0 \\
\hline 20 & 4 & 2 & 0 & 1 \\
\hline 21 & 83 & 28 & 5 & 6 \\
\hline 22 & 54 & 18 & 3 & 1 \\
\hline 23 & 40 & 15 & 2 & 1 \\
\hline 24 & 84 & 35 & 6 & 8 \\
\hline 25 & 71 & 26 & 3 & 2 \\
\hline 26 & 52 & 19 & 2 & 4 \\
\hline Total & 198 & 84 & 31 & 47 \\
\hline
\end{tabular}

la menor cantidad posible de unidades de protección (Villaseñor et al., 2003). Por otra parte, para evaluar la importancia de una determinada área en las estrategias de conservación, se han usado con frecuencia los criterios de la riqueza de especies y la 
magnitud del endemismo (Margules y Usher, 1981; Kirkpatrick, 1983; MacKinnon et al., 1990; Groombridge, 1992; McNeely, 1995; Lira et al., 2002; Villaseñor et al., 2003).

Para determinar los cuadros prioritarios para la potencial conservación de las especies de Asteraceae del DCH en Zacatecas, usando métodos iterativos, se evaluaron en primera instancia los cuadros considerados irreemplazables por contener especies raras y se utilizó el algoritmo propuesto por Margules et al. (1988), con las modificaciones hechas por Villaseñor et al. (2003), que permite identificar de manera jerárquica los cuadros importantes con base en un valor de rareza o endemismo (Kerr, 1997). Este atributo tiene la ventaja de tomar en cuenta la riqueza total de cada entidad, pero asignando un valor diferencial de las especies con base en su frecuencia de aparición en el conjunto de cuadros. El valor de rareza (VR) se calculó con la formula:

$$
\mathrm{VR}=\sum_{\mathrm{i}=1}^{\mathrm{S}} \frac{1}{\mathrm{Q}}
$$

donde $s$ es el número total de especies incluidas en el estudio y $Q$ es la cantidad de cuadros ocupados por cada especie.

El procedimiento constó de los siguientes pasos: (1) se calculó el inverso de la frecuencia de presencia de cada especie; (2) se obtuvo el VR por cuadro a partir de la suma de los valores calculados en el inciso anterior; (3) se eligió el cuadro que tuvo el VR más grande y las especies presentes en él se eliminaron de las fases subsecuentes del análisis; al conjunto de especies remanente se le denominó el complemento; (4) nuevamente se definió el cuadro con el valor de rareza mayor, con base en las especies del complemento; (5) cuando dos o más cuadros tuvieron el mismo valor de rareza, se eligió el que tuvo la mayor riqueza de especies endémicas de México; (6) en los casos en que persistió la coincidencia, se escogió el cuadro más cercano a alguno de los seleccionados previamente; (7) el procedimiento terminó cuando se logró la inclusión de todas las especies (Villaseñor et al., 2003).

\section{RESULTADOS}

De los 26 cuadros en que fue dividido el DCH en Zacatecas (Fig. 2), solamente en dos (3 y 17) no se registraron especies de Asteraceae; seguramente tal carencia se debe a que son cuadros con muy poca (menos de $25 \%$ ) superficie de terrenos bajo 
estudio, la cual además carece de suficientes vías de comunicación, lo que hace difícil la exploración botánica en su territorio.

Para Zacatecas se han registrado 348 especies de Asteraceae como elementos de las zonas áridas y semiáridas de México (Cuadro 1). Sin embargo, solamente se han documentado hasta la fecha 198 para el territorio que corresponde al $\mathrm{DCH}$; de esta cifra, 83 son especies endémicas de México. Por otra parte, 55 fueron definidas como raras (por registrarse únicamente en un solo cuadro), 22 de las cuales son restringidas en su distribución a México y nueve al DCH (siete de ellas también endémicas de México).

La riqueza de especies por cuadro varió de 1 (cuadro 2) a 84 (cuadro 24), con un promedio de 49.3. Los dos cuadros con mayor riqueza (21 y 24) se localizan en el sur del área de estudio, en la zona donde convergen las ecorregiones DCH y Piedemonte de la Sierra Madre Occidental (Anónimo, 2008), cerca de la ciudad de Zacatecas, capital del estado. Uno de ellos incluye en su territorio la Zona de Preservación Ecológica El Cedral, en el municipio de Ciudad Cuauhtémoc (cuadro 24).

El número de especies endémicas de México por cuadro varió de 2 (cuadro 20) a 35 (cuadro 24), con un promedio de 15.9 (Cuadro 2). Después del cuadro 24, los que registran la mayor cantidad de elementos endémicos son el 7 y 21 (cada uno con 28 especies) y el 25 (26 especies). Se observó una correlación estadística fuerte entre la riqueza total de especies y la de las endémicas por cuadro $(\mathrm{r}=0.953 ; \mathrm{P}<0.05)$. Los cuadros ubicados en el norte del área de estudio albergaron un mayor número de especies endémicas del DCH. En este sentido destacaron $\operatorname{los} 7$ y 12, cada uno con 13 elementos (Cuadro 2).

Tres plantas tuvieron la mayor frecuencia de presencia, al encontrarse en 22 de los 26 cuadros evaluados (Gymnosperma glutinosum (Spreng.) Less., Xanthisma spinulosum (Pursh) D.R. Morgan \& R.L. Hartm. y Zinnia acerosa (DC.) A. Gray). La especie endémica de México con área de distribución más amplia estuvo presente en 17 cuadros (Sanvitalia angustifolia Engelm. ex A. Gray) y la restringida al DCH con mayor frecuencia fue Chaetopappa bellioides (A. Gray) Shinners (10 cuadros). Varias de las especies consideradas como endémicas del DCH se conocen de partes de Zacatecas ubicadas fuera del límite de la ecorregión del DCH propuesta por INEGI (Anónimo, 2008; Fig. 1).

Las especies de Asteraceae registradas en la porción zacatecana del DCH son preponderantemente endémicas del territorio del país $(83,41.9 \%)$. Siguen en importancia las que se distribuyen de México hacia los Estados Unidos de América (70, $35.3 \%$ ); otras 19 especies (9.5\%) se reparten desde los Estados Unidos hasta Sudamérica. Un número relativamente bajo se distribuye de México a Centroamérica (14, 
Cuadro 3. Prioridades de conservación de los cuadros de 30' de latitud y longitud en que se dividió al estado de Zacatecas. La jerarquía de selección está basada en el análisis iterativo de las especies de Asteraceae registradas en el Desierto Chihuahuense (DCH). El complemento es el conjunto de especies remanente después de haber seleccionado el cuadro.

\begin{tabular}{cccc}
\hline Prioridad & Cuadro & Complemento & Acumulado(\%) \\
\hline 1 & 07 & 123 & $75(37.9)$ \\
2 & 24 & 68 & $130(65.7)$ \\
3 & 21 & 55 & $143(72.2)$ \\
4 & 26 & 47 & $151(76.3)$ \\
5 & 05 & 36 & $162(81.8)$ \\
6 & 14 & 28 & $170(85.9)$ \\
7 & 25 & 23 & $175(88.4)$ \\
8 & 12 & 17 & $181(91.4)$ \\
9 & 18 & 13 & $185(93.4)$ \\
10 & 06 & 10 & $188(94.9)$ \\
11 & 11 & 8 & $190(96.0)$ \\
12 & 16 & 6 & $192(97.0)$ \\
13 & 22 & 5 & $193(97.5)$ \\
14 & 23 & 4 & $194(98.0)$ \\
15 & 09 & 3 & $195(98.5)$ \\
16 & 01 & 2 & $196(99.0)$ \\
17 & 20 & 1 & $197(99.5)$ \\
18 & 04 & 1 & $198(100.0)$ \\
\hline
\end{tabular}

7\%) y otro mucho menor (dos especies) se conoce de México hasta Sudamérica. La distribución geográfica de otras tres se extiende al Viejo Mundo. A diferencia de éstas, los géneros mostraron un patrón diferente, pues de los 92 que incluyen las especies estudiadas, 31 se extienden hasta el Viejo Mundo (33.6\%), aunque también un porcentaje alto corresponde a los de área restringida a México y Estados Unidos $(25,27.1 \%)$. Solamente cuatro de ellos son endémicos de México y los demás presentan una distribución preferentemente hacia la porción sur del continente americano (Villaseñor, datos no publicados).

Como se indicó previamente, 55 especies fueron definidas como raras por haber sido registradas únicamente en un cuadro. Éstas se registraron en 17 de los 26, los cuales en conjunto incluyeron 197 de las 198 especies registradas en el DCH. 
Solamente Flaveria trinervia (Spreng.) C. Mohr no fue registrada en ninguno de estos 17 cuadros (conocida únicamente en el 4 y 19). Debido a que prácticamente toda la riqueza florística analizada estuvo incluida en 17 cuadros, el análisis iterativo únicamente se llevó a cabo con éstos, por considerarlos irreemplazables, añadiendo finalmente el 4, que de acuerdo con la metodología propuesta, se incluye para cubrir la potencial conservación de $100 \%$ de la flora evaluada.

El Cuadro 3 muestra los resultados del análisis iterativo. El cuadro 7 ocupó el primer lugar en la selección, lo que sugiere que su territorio debería ser considerado como prioritario para futuras estrategias de conservación de la diversidad florística del DCH en el estado. Otros cuadros importantes para este mismo propósito son los números 21, 24, 25 y 26, ubicados en el sur del área de estudio, los 5, 6 y 12 en el norte y 14 y 18 al oeste. El 5, identificado por el análisis iterativo en la quinta posición, incluye el Pico de Teyra, la única zona con alta prioridad para conservar la biodiversidad del DCH que ha sido identificada en el estado de Zacatecas (Anónimo, 2004). En conjunto, estos 10 cuadros contribuirían a proteger casi $95 \%$ de las especies de Asteraceae del DCH.

\section{DISCUSIÓN}

Puede afirmarse que las Asteraceae de la porción del DCH en Zacatecas están en general satisfactoriamente muestreadas. Los cuadros que carecen de datos o que contienen cifras bajas de especies, incluyen una parte muy pequeña del área de estudio, comparada con los que sí disponen de mejor información. Por otra parte, los cuadros donde se registran datos muestran cifras dispares; sin embargo, en la mayoría de ellos el número de especies es superior al promedio (ver Cuadro 2). Con esta información es posible ubicar con cierta confianza las áreas del estado que podrían contribuir potencialmente a la conservación de la diversidad florística del DCH, aceptando el papel relevante que Asteraceae representa en los estudios de biodiversidad en que ha sido utilizada como variable substituta de la diversidad florística de México (Rzedowski, 1991; Villaseñor et al., 2007).

El número de especies de Asteraceae registradas para la porción zacatecana del DCH equivale a $43 \%$ de las que se reportan para el estado. 150 especies adicionales de esta familia conocidas del estado han sido citadas como miembros del matorral xerófilo de otras partes de la República Mexicana. Algunas de éstas se reportan de la zona árida y semiárida de Zacatecas, pero fuera de los límites del DCH utilizados en este trabajo. Ejemplos de ellas son Astranthium orthopodum (B.L. Rob. \& Fer- 
nald) Larsen, Bartlettia scaposa A. Gray (Fig. 1), Bidens schaffneri (A. Gray) Sherff, Brickellia coulteri A. Gray o Coreopsis macvaughii D.J. Crawford, entre otras.

Varias especies endémicas del DCH también extienden su distribución geográfica a sitios no considerados dentro de los límites propuestos por INEGI (Anónimo, 2008). Tal es el caso de Gaillardia comosa A. Gray. Será necesario en un futuro revisar el contorno del DCH en Zacatecas, tomando en cuenta el área conocida de los organismos que de una u otra manera han sido utilizadas para definirlo. Por ejemplo, si Gaillardia comosa A. Gray es endémica del DCH, será necesario incluir los sitios fuera del polígono señalado por INEGI (Anónimo, 2008).

Los cuadros que poseen los mayores números de especies de Asteraceae (24 y 21) cubren una pequeña fracción del territorio del DCH. Fueron identificados por el análisis iterativo como segundo y tercer lugar en la jerarquía y destacan por varias razones. En primer lugar albergan la mayor riqueza conocida en la actualidad para el extremo sur del DCH; la flora de estos cuadros es una de las mejor conocidas del estado, porque su cercanía con la ciudad de Zacatecas ha facilitado su exploración botánica y en esta zona se ubica la ecotonía entre el DCH y otras ecorregiones, como el Piedemonte de la Sierra Madre Occidental y las porciones semiáridas del centro del país (Aguascalientes, Hidalgo, Jalisco y Querétaro). Especies características de la Sierra Madre Occidental, como Baccharis thesioides Kunth, Cosmos parviflorus (Jacq.) Pers., Hieracium abscissum Less., Pseudognaphalium brachypterum (DC.) Anderb., Roldana heracleifolia (Hemsl.) H. Rob. \& Brettell o Stevia lucida Lag. var. lucida, conviven en esta región con plantas típicas del DCH, como Bahia absinthifolia Benth., Flourensia cernua DC., Gymnosperma glutinosum (Spreng.) Less., Parthenium incanum Kunth, Zaluzania triloba (Ortega) Pers. o Zinnia acerosa (DC.) A. Gray. Es probable que tal combinación de especies sea resultado de las condiciones climáticas más húmedas y frescas que prevalecen en esta región. El cuadro que ocupa la tercera posición por riqueza de especies (7) y el adyacente (6) ubicado en la quinta posición, se localizan en la parte norte del área de estudio, en una región que se caracteriza por su topografía montañosa.

Los cuadros con más plantas endémicas de México son también los que tienen la mayor riqueza total. En este sentido destacan los 6, 7, 21 y 24 (ver Cuadro 2). Un patrón similar se ha observado a nivel nacional para las especies de Agavaceae (García-Mendoza, 1995) y Cucurbitaceae (Lira et al., 2002), pues sitios de alta diversidad de especies de esas familias son igualmente de elevada concentración de endemismo. Este patrón también se observa para los géneros de Asteraceae en la República Mexicana (Villaseñor et al., 1998) y para la flora del bosque tropical húmedo de México (Villaseñor et al., 2003). De acuerdo con Villaseñor et al. (2003), la aplicación prác- 
tica de esta combinación de riqueza y endemismo por cuadro es importante, porque permite seleccionar de forma simultánea áreas ricas en ambos aspectos.

Las plantas de Asteraceae endémicas del DCH son más abundantes en los cuadros ubicados en el norte del área de estudio. Los de los números 6,7 y 12 son los más ricos en especies con distribución geográfica restringida al DCH (Cuadro 2). La predominancia de endemismo en la parte norte del estado, probablemente esté asociada con los requerimientos edáficos específicos de algunos de estos taxones. Por ejemplo, Sartwellia mexicana A. Gray (Fig. 1) se desarrolla mejor en suelos yesosos, que en el estado de Zacatecas únicamente existen en este sector; sin embargo, Varilla mexicana A. Gray var. mexicana, adaptada a suelos salinos frecuentes en toda la zona árida y semiárida de Zacatecas, solamente ha sido registrada en el norte del estado (Johnston, 1974, Fig. 1). Es necesario considerar este patrón de rareza por factores edáficos en futuros esfuerzos de conservación de las especies exclusivas del DCH.

Las especies de Asteraceae cuyas áreas de distribución se extienden hacia los Estados Unidos representan un porcentaje mayor que aquellas que se distribuyen hacia Centro y Sudamérica. Estos datos no apoyan la ubicación del DCH como parte del Reino Neotropical como lo propone Rzedowski (1978). Más bien respaldan definiciones como la de Takhtajan (1986) o Morrone et al. (2002), que sitúan al DCH (o la zona árida y semiárida de la Altiplanicie Mexicana) como parte del Reino Holártico.

Los cuadros donde se registraron especies raras pueden contribuir a la conservación de la mayor cantidad de especies de Asteraceae, en el menor número posible de unidades de protección. En 17 de los 24 cuadros con información se localizan 99.5\% de las especies de Asteraceae del DCH. Sin embargo, la superficie que se requiere para conservar tal diversidad florística es, de esta manera, muy extensa, por el tamaño de los cuadros usados en el análisis iterativo. Es necesario extender el análisis a escalas más finas para realmente identificar los mejores sitios dentro de los cuadros ya seleccionados. Por ejemplo, en un ejercicio preliminar, en el que se evaluó la riqueza de especies de los cuadros definidos como importantes en el norte del área de estudio (1, 5, 6 y 7), dividiéndolos en cuadros de 6 ' de latitud y 6 'de longitud, se encontró que de los 100 así generados, solamente 64 incluyen parte de la porción zacatecana del DCH. De este número, 12 serían suficientes para incluir $100 \%$ de la riqueza de Asteraceae del norte del área de estudio (113 especies). Tal conjunto representa una extensión de alrededor de $1200 \mathrm{~km}^{2}$ (120,000 hectáreas), superficie que resulta más manejable para fines prácticos que los $10,000 \mathrm{~km}^{2}$ que suman los cuatro cuadros de 30' de latitud y longitud que los incluyen. 
El estado de Zacatecas puede ayudar en la conservación de alrededor de 51\% de las Asteraceae y de 46\% de la flora vascular del DCH. La riqueza y el endemismo de la flora xerofítica de Zacatecas destacan de manera especial por el número de especies que encuentran su límite de distribución en el estado. En el norte y el noroeste de Zacatecas se ubica el límite meridional de la distribución de al menos 10\% de las especies de Asteraceae endémicas del DCH. Finalmente, aunque Zacatecas alberga un número de especies menor que los otros estados que constituyen el DCH (Cuadro 1), su flora es significativamente distinta de la encontrada en San Luis Potosí. La particular riqueza de estos dos estados que delimitan la franja meridional del $\mathrm{DCH}$, justificaría llevar a cabo estrategias de conservación en ambos, no únicamente haciendo énfasis en San Luis Potosí, como se ha discutido en la literatura relacionada con este tema (Anónimo, 2004; Hernández y Bárcenas, 1996).

\section{AGRADECIMIENTOS}

Este trabajo forma parte de la tesis doctoral del primer autor y su realización fue posible gracias al apoyo económico que para el estudio de la biogeografía y conservación de las Asteraceae en el estado de Zacatecas fue otorgado por el Consejo Nacional de Ciencia y Tecnología (28575N). Agradecemos a Enrique Ortiz del instituto de Biología de la Universidad Nacional Autónoma de México (UNAM) su asesoría en el manejo de la cartografía digital, en el uso del sistema de información geográfica así como en la elaboración de la fig. 2 y a Rosario Redonda-Martínez, de la misma institución, su asistencia técnica para la elaboración del los trazos biogeográficos de varias especies endémicas del Desierto Chihuahuense y la elaboración de la fig. 1. Agradecemos también a David Enríquez Enríquez, de la Unidad Académica de Agronomía de la Universidad Autónoma de Zacatecas y a Guillermo IbarraManríquez, del Centro de Investigación en Ecosistemas de la UNAM, la lectura crítica del manuscrito y sus valiosas observaciones.

\section{LITERATURA CITADA}

Anónimo. 1981. Síntesis geográfica de Zacatecas. Coordinación General de los Servicios Nacionales de Estadística, Geografía e Informática. Secretaría de Programación y Presupuesto. México, D.F. 222 pp.

Anónimo. 1999. Ecorregiones de México, escala 1: 1 000,000. Comisión Nacional para el Conocimiento y Uso de la Biodiversidad. México, D.F. 
Anónimo. 2004. Valoración ecorregional para la conservación del Desierto Chihuahuense. 2a. edición. Pronatura Noreste, The Nature Conservancy y World Wildlife Fund. México, D.F. 105 pp.

Anónimo. 2008. Ecorregiones terrestres de México, escala 1:1 000 000. Instituto Nacional de Estadística, Geografía e Informática, Comisión Nacional para el Conocimiento y Uso de la Biodiversidad e Instituto Nacional de Ecología. México, D.F.

Anónimo. 2009. Sistema nacional de áreas naturales protegidas. Comisión Nacional de Áreas Naturales Protegidas. Secretaría de Medio Ambiente y Recursos Naturales. http://www.conanp.gob.mx/que_hacemos/sinap.php

Balleza, J. J. y J. L. Villaseñor. 2002. La familia Asteraceae en el estado de Zacatecas (México). Acta Bot. Mex. 59: 5-69.

Balleza, J. J., J. L. Villaseñor y G. Ibarra-Manríquez. 2005. Regionalización biogeográfica de Zacatecas, México, con base en los patrones de distribución de la familia Asteraceae. Rev. Mex. Biodiv. 76: 71-78.

Contreras, A. 1955. Definición de las zonas áridas y su delimitación en la República Mexicana. In: Beltrán, E. (ed.). Problemas de las zonas áridas de México. Instituto Mexicano de Recursos Naturales Renovables. México, D.F. pp. 3-40.

Enríquez, E. D., S. D. Koch y M. S. González-Elizondo. 2003. Flora y vegetación de la Sierra de Órganos, municipio de Sombrerete, Zacatecas, México. Acta Bot. Mex. 64: 45-89.

Ferrier, S., R. L. Pressey y T. W. Barret. 2000. A new predictor of the irreplaceability of areas for achieving a conservation goal, its application to real-world planning, and a research agenda for further refinement. Biol. Conserv. 93: 303-325.

Ferrusquía-Villafranca, I. 1990. Regionalización biogeográfica. Mapa IV.8.10. Atlas Nacional de México, vol. III. Instituto de Geografía, Universidad Nacional Autónoma de México. México, D.F.

García, E. 1987. Modificaciones al sistema de clasificación climática de Köppen. 4a. Edición particular. México, D.F. 217 pp.

García-Mendoza, A. 1995. Riqueza y endemismos de la familia Agavaceae en México. In: Linares, E., P. Dávila, F. Chiang, R. Bye y T. S. Elias (eds.). Conservación de plantas en peligro de extinción: diferentes enfoques. Instituto de Biología. Universidad Nacional Autónoma de México. México, D.F. pp. 51-75.

Groombridge, B. (ed.). 1992. Global biodiversity: status of the Earth's living resource. World Conservation Monitoring Centre. Chapman \& Hall. Londres. 614 pp.

Henrickson, J. y M. C. Johnston. Inédito. A flora of the Chihuahuan Desert Region. Borrador para publicación.

Hernández, H. M. y R. T. Bárcenas. 1996. Endangered cacti in the Chihuahuan Desert: II. Biogeography and conservation. Conserv. Biol. 10: 1200-1209.

Hernández, H. M., C. Gómez-Hinostrosa y B. Goettsch. 2004. Checklist of Chihuahuan desert Cactaceae. Harvard Pap. Bot. 9: 51-68.

Johnston, M. C. 1974. Brief resume of botanical, including vegetational, features of the Chihuahuan Desert Region with special emphasis on their uniqueness. In: Wauer, R. H. y D. H. Riskind (eds.). Transactions of the symposium on the biological resources of the Chihuahuan Desert Region, United States and Mexico. Sul Ross State University. Alpine, Texas. pp. 335-359. 
Kerr, J. T. 1997. Species richness, endemism, and the choice of areas for conservation. Conserv. Biol. 11: 1094-1100.

Kirkpatrick, J. B. 1983. An iterative method for establishing priorities for the selection of nature reserves: an example from Tasmania. Biol. Conserv. 25: 127-134.

Lira, R., J. L. Villaseñor y E. Ortíz. 2002. A proposal for the conservation of the family Cucurbitaceae in México. Biodivers. and Conserv. 11: 1699-1720.

MacKinnon, J., K. MacKinnon, G. Child y J. Thorsell (comps.). 1990. Manejo de áreas protegidas en los trópicos. Unión Internacional para la Conservación de la Naturaleza y los Recursos Naturales y Programa de las Naciones Unidas para el Medio Ambiente. Gland. 314 pp.

Margules, C. R. y M. B. Usher. 1981. Criteria used in assessing wildlife conservation potential: a review. Biol. Conserv. 21: 79-109.

Margules, C. R., A. O. Nicholls y R. L. Pressey. 1988. Selecting networks of reserves to maximise biological diversity. Biol. Conserv. 43: 63-76.

Margules, C. R. y J. L. Stein. 1989. Patterns in the distributions of species and the selection of nature reserves: an examples from Eucalyptus forest in South-eastern New South Wales. Biol. Conserv. 50: 219-238.

Margules, C. R., I. D. Cresswell y A. O. Nichols. 1994. A scientific basis for establishing networks of protected areas. In: Forey, P. L., C. J. Humphries y I. Vane Wright (eds.). Systematics and conservation evaluation, Systematics Association Special Volume 50. Claredond Press. Oxford. pp. 327-350.

Margules, C. R. y R. L. Pressey. 2000. Systematic conservation planning. Nature 403: 243253.

Mcneely, J. A. 1995. Keep all the pieces: systematics 2000 and world conservation. Biol. Conserv. 4: 510-519.

Medellín-Leal, F. 1982. The Chihuahuan Desert. In: Bender, G. L. (ed.). Reference handbook on the deserts of North America. Greenwood Press. Westport, Connecticut. pp. 321372 .

Morafka, D. J. 1977. A biogeographical analysis of the Chihuahuan Desert through its herpetofauna. Dr. W. Junk B.V. Publishers, The Hague. 313 pp.

Morrone, J. J., D. Espinosa O. y J. Llorente B. 2002. Mexican biogeographic provinces: preliminary scheme, general characterizations, and synonymies. Acta Zool. Mex. (número especial) 85: 83-108.

Nichols, A. O. y C. R. Margules. 1993. An upgraded reserve selection algorithm. Biol. Conserv. 64: 161-165.

Powell, A. M. y B. L. Turner. 1974. Aspects of the plant biology of the gypsum outcrops of the Chihuahuan Desert. In: Wauer, R. H. y D. H. Riskind (eds.). Transactions of the symposium on the biological resources of the Chihuahuan Desert Region, United States and Mexico. Sul Ross State University. Alpine, Texas. pp. 315-325.

Pressey, R. L. y A. O. Nichols. 1989a. Efficiency in conservation evaluation: scoring versus iterative approaches. Biol. Conserv. 50: 199-218. 
Pressey, R. L. y A. O. Nichols. 1989b. Applications of numerical algorithm to the selection of reserves in semi-arid New South Wales. Biol. Conserv. 50: 263-278.

Pressey, R. L., M. Bedward y D. A. Keith. 1994. New procedures for reserve selection in New South Wales: maximizing the chances of achieving a representative network. In: Forey, P. L., C. J. Humphries y I. Vane-Wright (eds.). Systematics and conservation evaluation. Systematics Associations Special Volume 50. Claredon Press. Oxford. pp. 351-373.

Rebelo, A. G. y W. R. Siegfried. 1992. Where should nature reserves be located in the Cape Floristic Region, South Africa? models for the spatial configuration of a reserves network aimed at maximizing the protection of flora diversity. Conserv. Biol. 6: 243252.

Rebelo, A. G. 1994. Iterative selection procedures: centres of endemism and optimal placement of reserves. Strelitzia 1: 231257.

Rzedowski, J. 1957. Vegetación de las partes áridas de los estados de San Luis Potosí y Zacatecas. Rev. Soc. Mex. Hist. Nat. 18: 49-101.

Rzedowski, J. 1972. Contribuciones a la fitogeografía florística de México. III. Algunas tendencias en la distribución geográfica y ecológica de las Compositae mexicanas. Ciencia (México) 27: 123-132.

Rzedowski, J. 1978. Vegetación de México. Limusa. México, D.F. 432 pp.

Rzedowski, J. 1991. Diversidad y orígenes de la flora fanerogámica de México. Act. Bot. Mex. 14: 3-21.

Schmidt, R. H. 1979. A climatic delineation of the "real" Chihuahuan Desert. J. Arid Environm 2: 243-250.

Shreve, F. 1942. The desert vegetation of North America. Bot. Rev. 8: 195-246.

Takhtajan, A. 1986. Floristic regions of the world. University of California Press. Berkeley and Los Angeles, California. 522 pp.

Toledo, V. M. y M. A. Ordóñez. 1998. El panorama de la biodiversidad de México: una revisión de los hábitats terrestres. In: Ramamoorthy, T. P., R. Bye, A. Lot y J. Fa. (eds.). Diversidad biológica de México: orígenes y distribución. Universidad Nacional Autónoma de México. México, D.F. pp. 739-757.

Turpie, J. K. 1995. Prioritizing South African estuaries for conservation: a practical example using waterbirds. Biol. Conserv. 74: 175-185.

Van Devender (inédito). Introducción. In: Henrickson, J. y M. C. Johnston. (inédito). A flora of the Chihuahuan Desert Region. Borrador para publicación.

Villaseñor, J. L. 1993. La familia Asteraceae en México. In: Gío-Argáez, R. y E. LópezOchoterena (eds.). Diversidad biológica de México. Rev. Soc. Mex. Hist. Nat. (número especial) 44: 117-124.

Villaseñor, J. L., G. Ibarra-Manríquez y D. Ocaña. 1998. Strategies for the conservation of Asteraceae in Mexico. Conserv. Biol. 12: 1066-1075.

Villaseñor, J. L., J. A. Meave, E. Ortiz y G. Ibarra-Manríquez. 2003. Biogeografía y conservación de los bosques tropicales húmedos de México. In: Morrone, J. J. y J. Llorente (eds.). 2003. Una perspectiva latinoamericana de la biogeografía. Universidad Nacional Autónoma de México. México, D.F. pp. 209-216. 
Villaseñor, J. L., P. Maeda, J. A. Rosell y E. Ortiz. 2007. Plant families as predictors of plant biodiversity in Mexico. Divers. Distrib. 13: 871-876.

Villaseñor, J. L., E. Ortiz y R. Redonda-Martínez. 2008. Catálogo de autores de plantas vasculares de México. 2a ed. Universidad Nacional Autónoma de México. México, D.F. 69 pp.

Wiggins, I. L. 1964. Flora of the Sonoran Desert. In: Shreve, F. \& I. L. Wiggins. Vegetation and flora of the Sonoran Desert. Stanford University Press. Stanford, California. 2 vols. 


\section{ANEXO}

Especies de Asteraceae en la porción del Desierto Chihuahuense en Zacatecas. Se indican los cuadros donde han sido registradas y a continuación se menciona su distribución a nivel mundial. Los autores de los nombres de las especies se citan con base en Villaseñor et al. (2008). El asterisco indica que la especie es endémica del Desierto Chihuahuense.

Acourtia fruticosa (La Llave) B.L. Turner; 09, 24; endémica de México Acourtia mexicana (Lag. ex D. Don) H. Rob.; 21, 24; endémica de México.

Acourtia nana (A. Gray) Reveal \& R.M. King; 01, 05, 07, 11, 12, 14, 16, 20, 21, 22, 23, 26;

México a Estados Unidos de América.

Acourtia parryi (A. Gray) Reveal \& R.M. King; 05, 06, 11, 12, 14, 16, 18, 26; endémica de México.

Acourtia wrightii (A. Gray) Reveal \& R.M. King; 01, 12, 14, 25; México a Estados Unidos de América.

*Acourtia zacatecana B.L. Turner; 24; endémica de México.

Adenophyllum cancellatum (Cass.) Villarreal; 24, 25; endémica de México. *Ageratina astellera (B.L. Turner) B.L. Turner; 06, 07; endémica de México. Ageratina brevipes (DC.) R.M. King \& H. Rob.; 21, 24; endémica de México.

Ageratina calaminthifolia (Kunth) R.M. King \& H. Rob.; 14, 24; endémica de México. Ageratina calophylla (Greene) R.M. King \& H. Rob.; 06; endémica de México. *Ageratina campyloclada (B.L. Rob.) R.M. King \& H. Rob.; 07; endémica de México. Ageratina espinosarum (A. Gray) R.M. King \& H. Rob.; 05, 21, 22, 24, 25, 26; endémica de México. Ageratina petiolaris (Moç. \& Sessé ex DC.) R.M. King \& H. Rob.; 07, 22, 24, 26; endémica de México.

Ageratina scorodonioides (A. Gray) R.M. King \& H. Rob.; 05, 06, 07, 21, 22, 23, 25, 26; endémica de México.

Ageratum corymbosum Zuccagni ex Pers.; 07, 21, 22, 24, 25, 26; Estados Unidos de América a Sudamérica.

Ambrosia canescens A. Gray; 01, 21, 24, 26; endémica de México.

Ambrosia confertiflora DC.; 06, 11, 12, 14, 16, 18, 21, 22, 23, 24, 25; México a Estados Unidos de América.

Ambrosia psilostachya DC.; 11, 26; México a Estados Unidos de América.

Aphanostephus ramosissimus DC.; 09, 12, 14, 16, 18, 21, 22, 24, 25, 26; endémica de México. Artemisia dracunculus L.; 11; México a Estados Unidos de América. Artemisia klotzschiana Besser; 05, 11, 21; endémica de México.

Artemisia ludoviciana Nutt.; 07, 11, 14, 16, 18, 21, 24, 25; Estados Unidos de América a Sudamérica.

*Baccharis neglecta Britton; 07; México a Estados Unidos de América.

Baccharis pteronioides DC.; 06, 12, 16, 18, 22, 24, 25, 26; México a Estados Unidos de América. 
Baccharis salicifolia (Ruiz \& Pav.) Pers.; 06, 07, 14, 22, 23, 24, 25, 26; amplia distribución en el Continente Americano.

Baccharis sulcata DC.; 06, 07, 25, 26; endémica de México.

Baccharis thesioides Kunth; 21; México a Estados Unidos de América.

Bahia absinthifolia Benth.; 01, 06, 07, 09, 11, 12, 14, 16, 18, 21, 22, 23, 24, 25; México a Estados Unidos de América.

Bahia glandulosa Greenm.; 22, 25; endémica de México

Bahia schaffneri S. Watson; 05, 12, 14, 16, 18, 21, 22, 23, 24, 25, 26; endémica de México.

Baileya multiradiata Harv. \& A. Gray ex Torr.; 18; México a Estados Unidos de América.

Barkleyanthus salicifolius (Kunth) H. Rob. \& Brettell; 01, 05, 06, 07, 11, 14, 16, 18, 21, 22, 23, 24, 25, 26; Estados Unidos de América a Sudamérica.

Berlandiera lyrata Benth.; 21, 24; México a Estados Unidos de América.

Bidens ferulifolia (Jacq.) DC.; 06, 24, 26; México a Estados Unidos de América.

Bidens odorata Cav.; 05, 06, 07, 11, 12, 14, 16, 18, 21, 22, 24, 25, 26; Estados Unidos de América a Sudamérica.

Bidens pilosa L.; 24; amplia distribución en el Continente Americano.

Brickellia eupatorioides (L.) Shinners; 05, 06, 12, 14, 16, 18, 21, 22; México a Estados Unidos de América.

*Brickellia laciniata A. Gray; 06, 07, 11, 12, 14, 16; México a Estados Unidos de América.

*Brickellia robinsoniana S.F. Blake; 14; endémica de México.

Brickellia secundiflora (Lag.) A. Gray; 05, 25; endémica de México.

*Brickellia spinulosa (A. Gray) A. Gray; 09, 24; endémica de México.

Brickellia subuligera (Schauer) B.L. Turner; 09, 24; endémica de México.

*Brickellia vernicosa B.L. Rob.; 24; endémica de México.

Brickellia veronicifolia (Kunth) A. Gray; 05, 06, 07, 09, 11, 12, 14, 16, 18, 21, 22, 24, 25 ;

México a Estados Unidos de América.

*Calanticaria brevifolia (Greenm.) E.E. Schill. \& Panero; 05, 07; endémica de México.

Calanticaria greggii (A. Gray) E.E. Schill. \& Panero; 12; endémica de México.

Calyptocarpus vialis Less.; 01, 05, 09; Estados Unidos de América a Sudamérica.

*Chaetopappa bellioides (A. Gray) Shinners; 05, 07, 11, 12, 14, 16, 21, 23, 25, 26; México a

Estados Unidos de América.

Chaetopappa ericoides (Torr.) G.L. Nesom; 06, 14, 21, 24, 25; México a Estados Unidos de América.

Chloracantha spinosa (Benth.) G.L. Nesom; 09; Estados Unidos de América a Sudamérica.

Chrysactinia mexicana A. Gray; 05, 06, 07, 11, 16, 21, 23, 26; México a Estados Unidos de América.

Cirsium mexicanum DC.; 06, 07, 11, 12, 16; endémica de México.

*Cirsium pringlei (S. Watson) Petr.; 12; endémica de México.

Cirsium rhaphilepis (Hemsl.) Petr.; 05, 06; endémica de México.

Conoclinium dissectum A. Gray; 01, 05, 06, 09, 12, 14, 16; México a Estados Unidos de América.

Conyza microcephala Hemsl.; 23; endémica de México.

Cosmos bipinnatus Cav.; 24; Estados Unidos de América a Sudamérica, naturalizada en el Viejo Mundo. 
Cosmos parviflorus (Jacq.) Pers.; 21; México a Estados Unidos de América.

Dahlia tubulata P.D. Sorensen; 07; endémica de México.

Dicranocarpus parviflorus A. Gray; 01, 06, 07, 12; México a Estados Unidos de América.

Dyssodia papposa (Vent.) Hitchc.; 01, 05, 06, 07, 11, 12, 14, 16, 18, 21, 22, 24, 25; Estados

Unidos de América a Sudamérica.

Dyssodia pinnata (Cav.) B.L. Rob.; 07, 16, 18, 21, 24; endémica de México.

Erigeron bigelovii A. Gray; 05; México a Estados Unidos de América.

Erigeron delphinifolius Willd.; 21, 26; México a Estados Unidos de América.

Erigeron flagellaris A. Gray; 07; México a Estados Unidos de América.

Erigeron janivultus G.L. Nesom; 07, 23; endémica de México.

Erigeron longipes DC.; 22; Estados Unidos de América a Sudamérica.

Erigeron modestus A. Gray; 07; México a Estados Unidos de América.

Erigeron pubescens Kunth; 05, 06, 07, 11, 16, 22, 23, 24, 25; endémica de México.

*Erigeron subacaulis (McVaugh) G.L. Nesom; 21, 24, 25; endémica de México.

Euphrosyne partheniifolia DC.; 01, 05, 07, 09, 12, 14; endémica de México.

Eutetras palmeri A. Gray; 25; endémica de México.

Flaveria anomala B.L. Rob.; 01, 06, 07, 11, 12; endémica de México.

Flaveria oppositifolia (DC.) Rydb.; 20; endémica de México.

Flaveria trinervia (Spreng.) C. Mohr; 04, 19; Nuevo y Viejo Mundo.

Fleischmannia pycnocephala (Less.) R.M. King \& H. Rob.; 11, 12, 16; amplia distribución en el Continente Americano.

Florestina pedata (Cav.) Cass.; 16; México a Centroamérica.

Flourensia cernua DC.; 01, 05, 06, 07, 11, 12, 14, 16, 21, 23; México a Estados Unidos de América.

*Flourensia dentata S.F. Blake; 06, 14, 18, 22; endémica de México.

* Gaillardia comosa A. Gray; 05, 06, 12, 14, 16, 18, 22, 25, 26; endémica de México.

Galinsoga parviflora Cav.; 05, 06, 07, 14, 21, 22, 23, 24, 25; Norteamérica al Viejo Mundo.

Gochnatia hypoleuca (DC.) A. Gray; 07; México a Estados Unidos de América.

*Grindelia oxylepis Greene; 11, 14, 21, 24; endémica de México.

Grindelia subdecurrens DC.; 07, 25; endémica de México.

*Gutierrezia conoidea (Hemsl.) M.A. Lane; 14, 15, 24; endémica de México.

Gutierrezia grandis S.F. Blake; 07; endémica de México.

Gutierrezia microcephala (DC.) A. Gray; 11; México a Estados Unidos de América.

Gutierrezia sarothrae (Pursh) Britton \& Rusby; 07; México a Estados Unidos de América.

Gutierrezia sericocarpa (A. Gray) M.A. Lane; 07, 09, 11, 18, 22; endémica de México.

Gutierrezia sphaerocephala A. Gray; 06; México a Estados Unidos de América.

Gymnosperma glutinosum (Spreng.) Less.; 01, 05, 06, 07, 09, 11, 12, 14, 16, 18, 20, 21, 22, 23,

24, 25, 26; Estados Unidos de América a Sudamérica.

Hedosyne ambrosiifolia (A. Gray) Strother; 07; México a Estados Unidos de América.

*Helenium elegans DC.; 12; endémica de México.

Helenium mexicanum Kunth; 01, 06, 11, 14, 18, 21, 22, 23, 24, 26; México a Centroamérica.

*Helenium microcephalum DC.; 01, 05, 09, 11, 12; México a Centroamérica.

Helianthus annuus L.; 06, 07, 12, 16, 18; Estados Unidos de América y México, naturalizada

en otras partes del mundo. 
Helianthus laciniatus A. Gray; 01, 05, 06, 07, 09, 12, 16, 18, 21, 22, 24; México a Estados Unidos de América.

Heterosperma pinnatum Cav.; 05, 06, 11, 12, 14, 16, 18, 21, 22, 23, 24, 25, 26; Estados Unidos de América a Sudamérica.

Heterotheca inuloides Cass.; 07, 12, 14, 16, 18, 21, 24; endémica de México.

*Heterotheca mucronata Harms ex B.L. Turner; 07, 12; endémica de México.

Heterotheca subaxilaris (Lam.) Britton \& Rusby; 21, 22; México a Estados Unidos de América.

Hieracium abscissum Less.; 26; Estados Unidos de América a Sudamérica.

Hybridella globosa (Ortega) Cass.; 23, 24; endémica de México.

Hymenoxys odorata DC.; 11, 23, 25; México a Estados Unidos de América.

Isocoma hartwegii (A. Gray) Greene; 22, 26; México a Estados Unidos de América.

Isocoma veneta (Kunth) Greene; 07, 12, 14, 18, 21, 22, 23, 24, 25, 26; endémica de México.

*Jefea brevifolia (A. Gray) Strother; 05, 07, 12; México a Estados Unidos de América.

Koanophyllon solidaginifolium (A. Gray) R. M. King \& H. Rob.; 05; México a Estados Unidos de América.

Laennecia confusa (Cronquist) G.L. Nesom; 21, 23, 24, 25; México a Centroamérica.

Laennecia coulteri (A. Gray) G.L. Nesom; 07, 09, 12, 14, 16, 23; México a Estados Unidos de América.

*Laennecia microglossa (S.F. Blake) G.L. Nesom; 18, 21; endémica de México.

Laennecia schiedeana (Less.) G.L. Nesom; 11, 18; Estados Unidos de América a Sudamérica.

Laennecia sophiifolia (Kunth) G.L. Nesom; 14, 18, 21, 22, 24, 25, 26; amplia distribución en el Continente Americano.

Leuciva dealbata (A. Gray) Rydb.; 09, 11, 12, 14, 16; México a Estados Unidos de América.

Machaeranthera tanacetifolia (Kunth) Nees; 06, 07, 09, 12, 14, 18, 21, 22, 23, 24; México a

Estados Unidos de América.

*Medranoa parrasana (S.F. Blake) Urbatsch \& R.P. Roberts; 05, 06; endémica de México.

Melampodium sericeum Lag.; 14; Estados Unidos de América a Sudamérica.

Montanoa leucantha (Lag.) S.F. Blake; 21, 24, 25; endémica de México.

*Nicolletia edwardsii A. Gray; 01, 07, 09, 12; México a Estados Unidos de América.

Parthenium argentatum A. Gray; 05, 06, 07, 11, 12, 16, 18; México a Estados Unidos de América.

Parthenium bipinnatifidum (Ortega) Rollins; 06, 09, 11, 12, 14, 16, 18, 21, 22, 23, 24, 25, 26; México a Estados Unidos de América.

Parthenium confertum A. Gray; 01, 06, 11, 12, 16; México a Estados Unidos de América.

Parthenium incanum Kunth; 01, 05, 06, 07, 09, 11, 12, 14, 16, 21, 22, 23, 24, 25, 26; México a Estados Unidos de América.

Pectis angustifolia Torr.; 14; México a Estados Unidos de América.

Pectis prostrata Cav.; 24; amplia distribución en el Continente Americano.

Perymenium mendezii DC.; 21, 23, 24, 25, 26; endémica de México.

Pinaropappus roseus (Less.) Less.; 05, 06, 07, 21, 24, 25, 26; México a Estados Unidos de América. 
Piqueria trinervia Cav.; 18, 21, 22, 23, 24, 25, 26; México a Centroamérica.

Pittocaulon praecox (Cav.) H. Rob. \& Brettell; 21, 24, 25; endémica de México.

Porophyllum scoparium A. Gray; 07, 18; México a Estados Unidos de América.

Pseudognaphalium attenuatum (DC.) Anderb.; 21, 25; México a Centroamérica.

Pseudognaphalium brachypterum (DC.) Anderb.; 21; México a Centroamérica.

Pseudognaphalium canescens (DC.) Anderb.; 21; México a Estados Unidos de América.

Pseudognaphalium conoideum (Kunth) Anderb.; 18; endémica de México.

Pseudognaphalium inornatum (DC.) Anderb.; 14; endémica de México.

Pseudognaphalium semiamplexicaule (DC.) Anderb.; 21; México a Centroamérica.

Pseudognaphalium viscosum (Kunth) Anderb.; 26; Estados Unidos de América a Sudamérica.

Psilactis brevilingulata Sch. Bip. ex Hemsl.; 06, 11, 14, 16, 18, 21, 22, 24, 26; Estados Unidos de América a Sudamérica.

Psilostrophe gnaphalodes DC.; 05, 06, 07, 11, 12; México a Estados Unidos de América.

Ratibida columnifera (Nutt.) Wooton \& Standl.; 12, 16; México a Estados Unidos de América.

Roldana heracleifolia (Hemsl.) H. Rob. \& Brettell; 24; endémica de México.

Sanvitalia abertii A. Gray; 14; México a Estados Unidos de América.

Sanvitalia angustifolia Engelm. ex A. Gray; 01, 06, 07, 09, 11, 12, 14, 16, 21, 22, 23, 26; endémica de México.

Sanvitalia ocymoides DC.; 14; México a Estados Unidos de América.

Sanvitalia procumbens Lam.; 05, 09, 14, 16, 18, 21, 22, 23, 24, 25, 26; México a Centroamérica.

*Sartwellia mexicana A. Gray; 06, 07, 11; endémica de México.

* Sartwellia puberula Rydb.; 01; endémica de México.

Schkuhria pinnata (Lam.) Kuntze; 14, 18, 21, 24, 25; México a Centroamérica.

Senecio flaccidus Less.; 06, 07, 14, 18, 21, 22, 24; México a Estados Unidos de América.

Senecio pseudopicridis T.M. Barkley; 07; endémica de México.

Simsia amplexicaulis (Cav.) Pers.; 06, 07, 11, 12, 14, 18, 21, 24, 25, 26; México a Centroamérica.

Solidago scabrida DC.; 05, 06, 07, 11, 12, 14, 16, 18, 21, 22, 25, 26; endémica de México.

Solidago velutina DC.; 16; México a Estados Unidos de América.

Stevia lucida Lag.; 21, 25; México a Sudamérica.

Stevia micrantha Lag.; 24, 25; México a Estados Unidos de América.

Stevia salicifolia Cav.; 07, 23, 25; México a Estados Unidos de América.

Stevia serrata Cav.; 21, 24, 25, 26; Estados Unidos de América a Sudamérica.

Stevia viscida Kunth; 24; Estados Unidos de América a Sudamérica.

Symphyotrichum expansum (Poepp. ex Spreng.) G.L. Nesom; 09, 22, 24, 25; México a

Centroamérica.

Tagetes lunulata Ortega; 21, 24, 25; endémica de México.

Tagetes micrantha Cav.; 21, 24, 25, 26; México a Estados Unidos de América.

Thelesperma longipes A. Gray; 07; México a Estados Unidos de América.

Thelesperma magapotamicum (Spreng.) Kuntze; 06, 07, 21, 24; México a Estados Unidos de América. 
Thymophylla acerosa (DC.) Strother; 05, 06, 07, 09, 11, 12, 14, 16, 18, 21, 22, 23, 24, 25, 26; México a Estados Unidos de América.

Thymophylla pentachaeta (DC.) Small; 05, 06, 07, 11, 12, 14, 16, 18, 21, 22, 24, 25, 26; Estados Unidos de América a Sudamérica.

Thymophylla setifolia Lag.; 05, 06, 07, 11, 12, 16, 18, 21, 22, 23, 24, 25, 26; México a Estados Unidos de América.

Thymophylla tenuifolia (Cass.) Rydb.; 01, 05, 06, 07, 12, 14, 16, 20, 21, 23, 26; endémica de México.

Tithonia tubiformis (Jacq.) Cass.; 01, 05, 06, 11, 12, 14, 18, 21, 22, 24, 25; México hasta Sudamérica.

Townsendia mexicana A. Gray; 05, 06, 11, 18, 21, 23, 24, 26; endémica de México.

Tridax balbisioides (Kunth) A. Gray; 14, 21, 24, 25; endémica de México.

Tridax coronopifolia (Kunth) Hemsl.; 22, 25; endémica de México.

Tridax palmeri A. Gray; 25; endémica de México.

Trixis angustifolia DC.; 06, 09, 11, 14, 18, 21, 22, 23, 24, 25, 26; endémica de México.

Trixis californica Kellogg; 01, 09, 11, 12; México a Estados Unidos de América.

*Varilla mexicana A. Gray; 01, 06; endémica de México.

Verbesina chihuahuensis A. Gray; 06, 07; endémica de México.

Verbesina encelioides (Cav.) Benth. \& Hook. f.; 01, 05, 06, 07, 09, 11, 12, 14, 18, 25, 26; México a Estados Unidos de América.

Verbesina longipes B.L. Rob. \& Greenm.; 01, 05, 06, 07, 09, 11, 12, 14, 16, 23; endémica de México.

Verbesina mollis Kunth; 26; endémica de México.

Verbesina pedunculosa (DC.) B.L. Rob.; 21; endémica de México.

Verbesina serrata Cav.; 09, 14, 21, 24, 25; endémica de México.

Verbesina virgata Cav.; 26; endémica de México.

Viguiera cordifolia A. Gray; 06, 11, 12, 14, 16, 18, 21, 24, 25; México a Estados Unidos de América.

Viguiera dentata (Cav.) Spreng.; 01, 05, 06, 07, 09, 14, 16, 18, 21, 22, 24, 25; México a Centroamérica.

Viguiera linearis (Cav.) Sch. Bip. ex Hemsl.; 14, 21, 22, 24, 25; endémica de México.

Viguiera stenoloba S.F. Blake; 05, 07, 11, 12, 16; México a Estados Unidos de América.

Xanthisma gymnocephalum (DC.) D.R. Morgan \& R.L. Hartm.;18; México a Estados Unidos de América.

Xanthisma spinulosum (Pursh) D.R. Morgan \& R.L. Hartm.; 01, 05, 06, 07, 09, 11, 12, 14, 16, 18, 21, 22, 23, 24, 25, 26; México a Estados Unidos de América.

Xanthium strumarium L.; 01, 09, 12, 14, 18, 21, 22, 24, 25; Nuevo y Viejo Mundo.

Xanthocephalum gymnospermoides (A. Gray) Benth.; 24; endémica de México.

Zaluzania augusta (Lag.) Sch. Bip.; 22, 24, 25; endémica de México.

*Zaluzania mollissima A. Gray; 05, 06, 07, 12, 21, 22, 23; endémica de México.

Zaluzania triloba (Ortega) Pers.; 06, 07, 11, 12, 14, 16, 18, 21, 23, 24, 25, 26; endémica de México.

Zinnia acerosa (DC.) A. Gray; 01, 05, 06, 07, 09, 11, 12, 14, 16, 18, 21, 22, 23, 24, 25, 26; México a Estados Unidos de América. 
Balleza y Villaseñor: Zacatecas en la conservación de la riqueza florística del Desierto Chihuahense

Zinnia angustifolia Kunth; 05, 11; endémica de México.

Zinnia juniperifolia (DC.) A. Gray; 06, 07, 12; endémica de México.

Zinnia peruviana (L.) L.; 09, 11, 14, 21, 24, 25; amplia distribución en el Continente Americano. 